\title{
Fow measurements of neutral strange and charged hadrons with the ALICE detector at LHC
}

\author{
Cristian Ivan* (for the ALICE Collaboration) \\ Research Division and ExtreMe Matter Institute EMMI, GSI Helmholtzzentrum für \\ Schwerionenforschung, Darmstadt, Germany \\ E-mail: cristian.george.ivan@cern.ch
}

\begin{abstract}
The azimuthal anisotropy of particles produced in heavy-ion collisions is one of the main observables to study collective behavior of matter under extreme conditions. We report the differential flow measurements of strange hadrons at mid-rapidity in $\mathrm{Pb}-\mathrm{Pb}$ collisions at $2.76 \mathrm{TeV}$ recorded with ALICE at the LHC. The elliptic and triangular flow for charged pions, kaons and protons measured by the ALICE collaboration are also shown and compared to hydrodynamical model calculations.
\end{abstract}

The Seventh Workshop on Particle Correlations and Femtoscopy

September 20 - 242011

University of Tokyo, Japan

\footnotetext{
* Speaker.
} 


\section{Introduction}

Anisotropic flow is an important observable for probing the properties of the hot and dense matter created in heavy-ion collisions. The first measurement of transverse momentum integrated elliptic flow $\left(v_{2}\right)$ at the LHC shows an increase of $30 \%$ with respect to the flow values measured at RHIC top energy although no significant change in the $\mathrm{p}_{\mathrm{t}}$ differential $v_{2}$ is ovserved [1]. These observations are attributed to the increase in the mean $\mathrm{p}_{\mathrm{t}}$. Since flow develops collective velocities, different hadron species will have different elliptic flow as a function of transverse momentum which gives rise to the mass splitting and mass ordering. Triangular flow is expected to show similar behavior as elliptic flow and its observation adds another constrain to the evaluation of the medium viscosity.

In this proceedings, the preliminary ALICE results on $v_{2}$ and $v_{3}$ of identified charged and neutral strange hadrons at the LHC are presented.

\section{Experimental setup}

The results presented in this proceedings were obtained from the data recorded with the ALICE detector in November 2010 during the first $\mathrm{Pb}-\mathrm{Pb}$ run at $\sqrt{s_{N N}}=2.76 \mathrm{TeV}$. The minimum bias trigger is provided by the VZERO counters and the silicon pixel detector (SPD). Standard event selections for $\mathrm{Pb}-\mathrm{Pb}$ collisions [1] together with a primary vertex position of $|z|<7 \mathrm{~cm}$ yield a sample of about 4 million events. The collision centrality is estimated using the VZERO scintillators which cover a rapidity range of $-3.7<\eta<-1.7$ and $2.8<\eta<5.1$. Tracking is done with the ALICE Inner Tracking System (ITS) and the Time Projection Chamber (TPC) which covers the full azimuth for $|\eta|<0.8$.

The identification of the charged hadrons is done by combining the information from the TimeOf-Flight detector (TOF) with the energy loss measured with the TPC. This results in a purity of more than $95 \%$ for pions and kaons at $p<3 \mathrm{GeV}$ and $p<5 \mathrm{GeV}$ for protons. Contamination from non-primary particles was reduced by requiring a distance of closest approach to the primary vertex of a maximum of $1 \mathrm{~mm}$. The main sources of systematic uncertainty on the flow of identified charged hadrons are non-flow feed-down and centrality determination.

Neutral strange hadrons were identified via their decay topology. A clean sample of $K_{s}^{0}$ and $\bar{\Lambda}$ is obtained by requiring that the decay products have at least 80 clusters in the TPC with $\left\langle\chi^{2}\right\rangle$ per TPC cluster $\leq 4$, distance of closest approach to the primary vertex of less than $1 \mathrm{~cm}$, impact parameter larger than $0.1 \mathrm{~cm}$ and a minimum transverse momentum of $0.2 \mathrm{GeV} / \mathrm{c}$.

Elliptic flow is measured using the two-particle scalar product method [2] with a large $\eta$ gap $(\Delta|\eta|>1)$ to reduce the contribution from short range non-flow correlations. A large systematic error of $10 \%$ for $K_{s}^{0}$ and $20 \%$ for $\bar{\Lambda}$ was considered due to the uncertainty on the $v_{2}$ efficiency as a function of the local track density. The uncertainties in the following figures indicate statistical and systematic errors added in quadrature. 


\section{Results}

Figure 1 shows the elliptic flow of identified charged particles for central (10\%-20\%) and midcentral (40\%-50\%) collisions. Theoretical predictions from viscous hydrodynamics with CGC initial conditions and viscosity to entropy ratio $\eta / s$ of $0.2[3]$ are shown as solid lines. To include effects of collective expansion from the hadronic phase, the model was extended with UrQMD calculations [4] which are shown as dashed lines.

As can be seen in the figure, mass splitting and mass ordering is present in the differential elliptic flow of pions, kaons and anti-protons. Pure hydrodynamical calculations agree reasonably well with the measured $v_{2}$ of charged pions and kaons for more central collisions (left panel) but start to deviate for $\mathrm{p}_{\mathrm{t}}>1.5 \mathrm{GeV} / \mathrm{c}$ in more peripheral collisions (right panel).

In central collisions, the elliptic flow of anti-protons is overpredicted at small transverse momentum. This is because the radial flow generated in the purely hydrodynamical model is not strong enough in central collisions to push the anti-proton elliptic flow to larger $\mathrm{p}_{\mathrm{t}}$. The fact that the $v_{2}$ of anti-protons is better described by theoretical calculations in more peripheral collisions might be due to an incorect description of the centrality dependence of the radial flow as compared to elliptic flow [4]. The hydrodynamical model extended with UrQMD describe the anti-protons better in central and mid-central collisions and an additional tuning of the $\eta / s$ is expected to describe all particle species in all centrality classes [4].

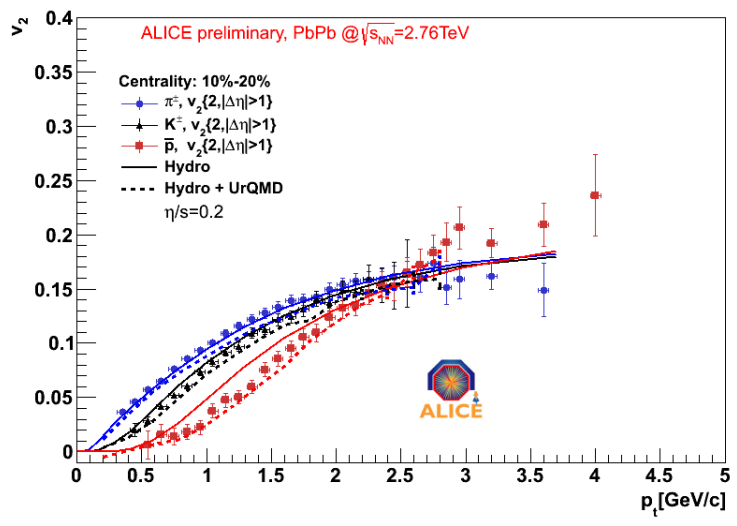

(a)

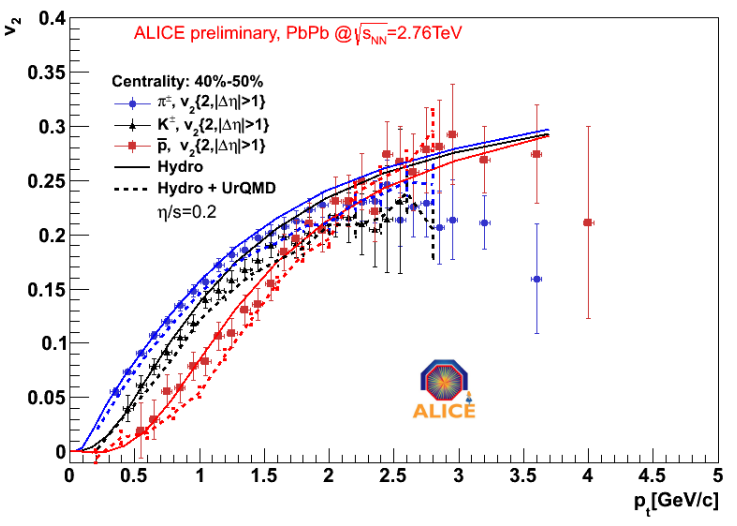

(b)

Figure 1: Identified charged particles elliptic flow for central (10\%-20\%) and mid-central (40\%$50 \%$ ) collisions compared to viscous hydrodynamic calculations [3] (solid lines) and [4] (dashed lines).

Figure 2 shows the elliptic flow for identified neutral hadrons compared to hydrodynamical calculations. A behavior similar to the charged identified particles is observed, i.e. mass splitting and mass ordering. For central collisions (10\%-20\%) elliptic flow of $\mathrm{K}_{s}^{0}$ agrees within experimental uncertainties with the theoretical calculations up to about $3.5 \mathrm{GeV} /$ in $\mathrm{p}_{\mathrm{t}}$ while for more peripheral collisions (40\%-50\%) only up to $1.5 \mathrm{GeV} / \mathrm{c}$. Similar to the anti-protons, the $\bar{\Lambda}$ is better described by hydrodynamical calculations for more peripheral collisions than for more central. 

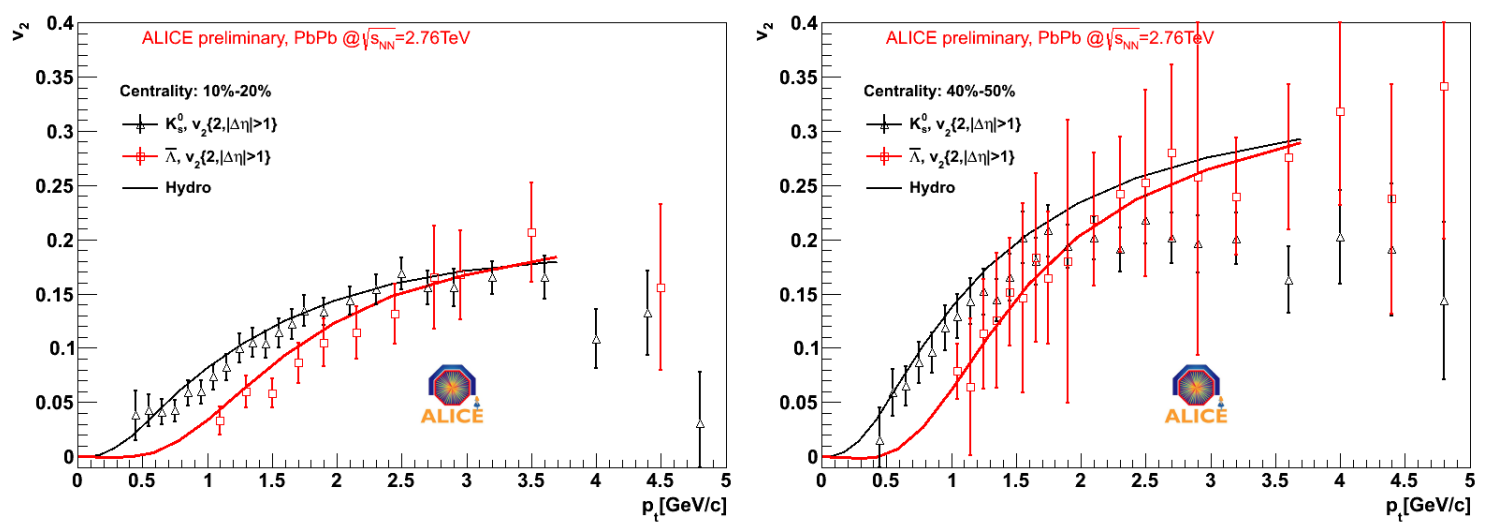

Figure 2: Neutral strange particles elliptic flow for central (10\%-20\%) and mid-central (40\%-50\%) collisions compared to hydrodynamic calculations [3].

Figure 3 shows the elliptic flow for identified charged and neutral particles scaled with the number of constituent quark versus the transverse kinetic energy per quark [5]. As can be seen, within experimental error bars, mesons follow the $K E_{t}$ scaling for both central and mid-central collisions. Baryons, on the other hand, deviate from quark scaling: stronger for central collisions (left panel) and less for mid-central collisions (right panel).
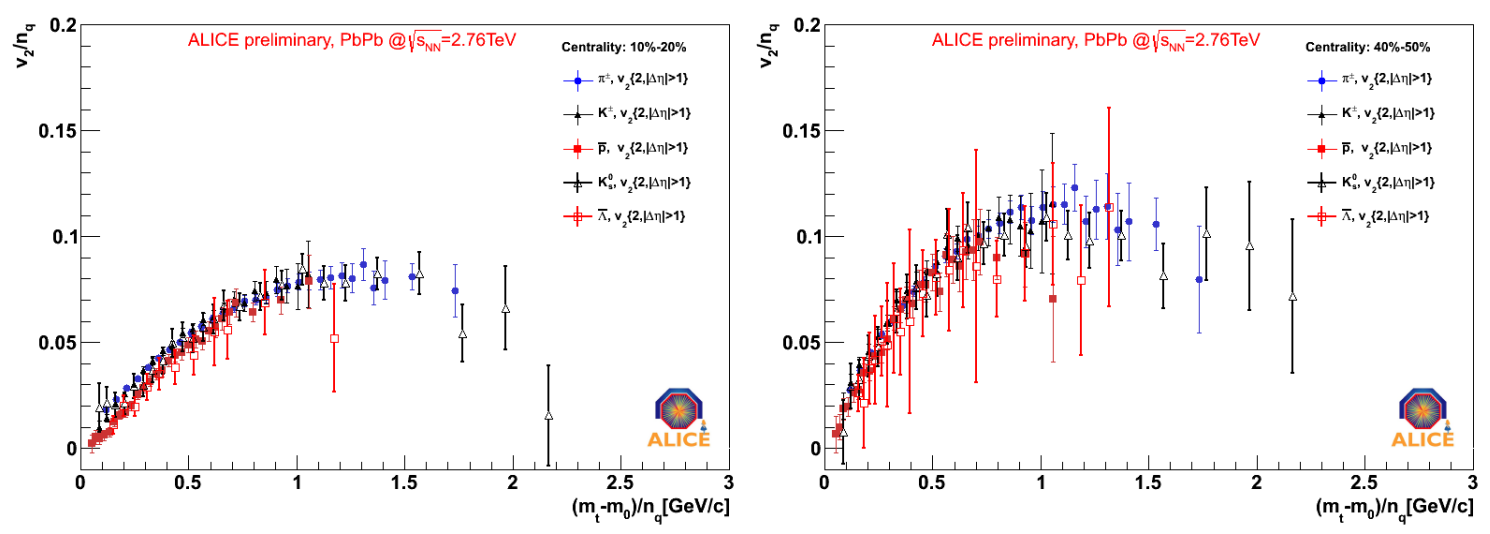

Figure 3: Elliptic flow per constituent quark versus transverse kinetic energy per quark for identified charged particles in central (left) and mid-central (right) collisions.

Figure 4 shows triangular flow for identified charged particles for central and mid-central collisions. A similar mass splitting and mass ordering as for elliptic flow is observed for low $\mathrm{p}_{\mathrm{t}}$. In contrast to elliptic flow, triangular flow does not show such a strong centrality dependence. Figure 5 shows deviations from the constituent quark scaling for both central and mid-central collisions. 

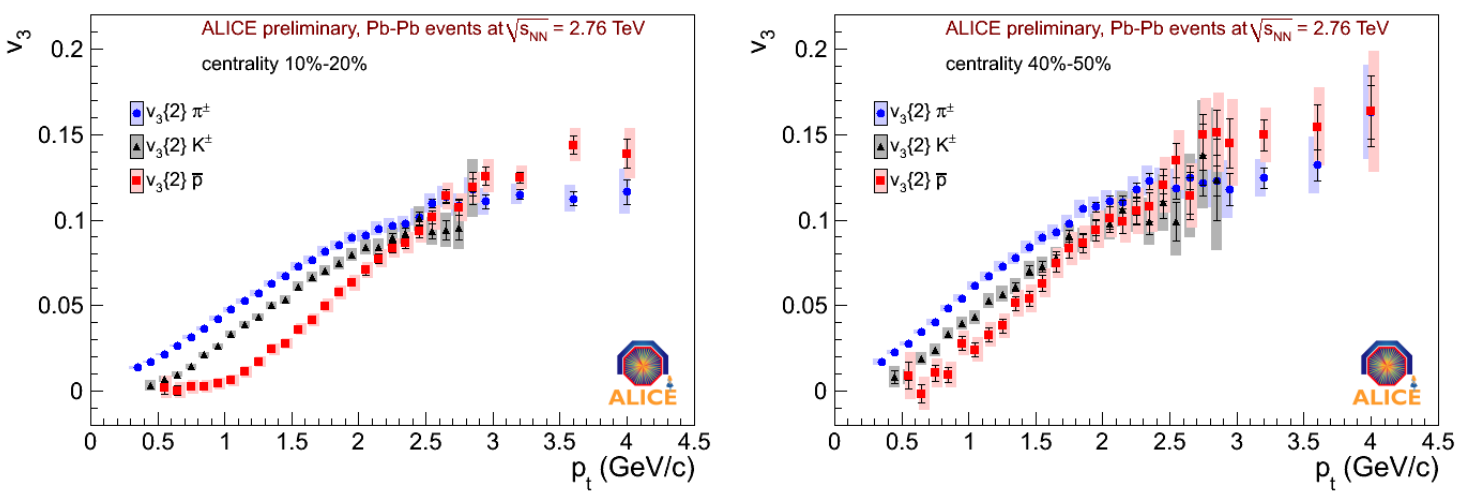

Figure 4: Triangular flow (scalar product method, without an $\eta$ gap) for identified charged particles in central (left) and mid-central (right) collisions.
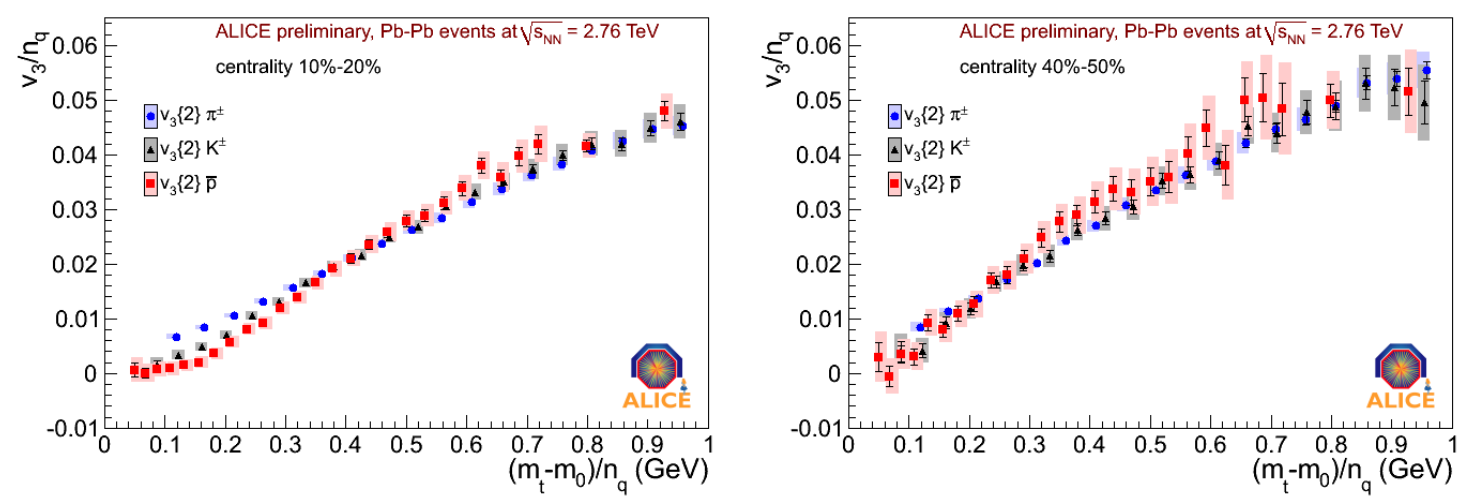

Figure 5: Constituent quark scaling of triangular flow (Fig. 4) for identified charged particles in central (left) and mid-central (right) collisions.

\section{Summary}

Measurements of $\mathrm{p}_{\mathrm{t}}$ differential elliptic and triangular flow are shown for $\pi^{ \pm}, \mathrm{K}^{ \pm}, \bar{p}, \mathrm{~K}_{s}^{0}$ and $\bar{\Lambda}$ in $\mathrm{Pb}-\mathrm{Pb}$ collisions at $\sqrt{s_{N N}}=2.76 \mathrm{TeV}$ and compared to hydrodynamic calculations. Data and pure hydrodynamic models agree reasonably well for pions and kaons in central collisions while for anti-protons the description holds better only for mid-central events. However, models which couple viscous hydrodynamics to a realistic model of the hadronic phase are expected to describe better the data at all centralities and for all particle species [4].

Elliptic and triangular flow show some common characteristics: mass splitting, mass ordering at low $\mathrm{p}_{\mathrm{t}}, K E_{t}$ scaling for mesons and deviations from this for baryons. 


\section{References}

[1] K. Aamodt et al (ALICE Collaboration), 2010 Phys. Rev. Lett. 105252302

[2] C. Adler et al (STAR Collaboration), 2002 Phys. Rev. C 66034904

[3] C. Shen, U.W. Heinz, P. Huovinen and H. Song, 2011 arXiv:1105.3226v1

[4] C. Shen, U.W. Heinz, P. Huovinen and H. Song, 2011 arXiv:1108.5323v1

[5] A. Adare et al (PHENIX collaboration), 2007 Phys. Rev. Lett. 98162301 